\title{
An experimental and theoretical Investigation into the flow through air box of a potato-rock separator
}

\author{
Jacob Bobby Sagayaraj \\ Akash Kushreshtha \\ Prabir Basu \\ Mechanical Engineering Department, Dalhousie University, Halifax \\ Lyle Gauthier \\ Allan Equipment, York, Prince Edward Island
}

\begin{abstract}
-
Velocity and pressure distribution in the air duct system and that over the grate of a potato-rock separator of Alan Equipment, PEI was analysed utilizing computational fluid dynamics. COMSOL software using appropriate boundary conditions was used for rapid analysis of flow through the system. To calibrate the theoretical results velocity, pressure and flow rates were measured on the actual full-scale unit. These tests were carried out using pitot tube, digital manometer and special duct flow measurer. Experimental result was compared with the obtained simulation result. The effect of duct geometry on the velocity distribution through the grate was established through this exercise. We make use of CFD-sofware to understand the pattern of the air flow in the air plenum for various designs. Thereafter different design options were analysed using CFD. The best option was chosen and was implemented in the modification of design of the full-seale rock potato separator. Experiment was carried out on the modified unit. The measurements showed good distribution of air as predicted by the CFD-simulation.
\end{abstract}

\section{INTRODUCTION}

Potato harvesting is a major agricultural activity of Prince Edward Island, where the need for faster yet efficient means of harvesting is of growing importance. Potatoes are harvested from the field by large machineries. Such mechanical means of digging potato from the ground naturally bring lots of rocks and dirt from the field. Separation of rocks from harvested potato is thus a critical step in this production cycle. Allan Equipment has developed a new type of machine to automatically separate rocks from potato, where an even distribution of air over a large grate of this machine is of prime importance for maximum efficiency of separation. The separator used was designed to aid in the separation of potatoes and rocks, which are harvested in the potato field. The potatoes and rocks are extracted from the field and sent into a conveyor. These potatoes and rocks along with the vines present in it fall onto a 'wave-bed' below which an air duct system is present. A centrifugal fan feeds air into the air plenum. The separation of potatoes from rock above the wave bed is dependent on various factors like shape and density of rock particles. The separation is achieved by the high flow of air, resulting in floatation of the potatoes. This high flow of air results in the levitation of potatoes and eventual removal through the exit because of the wave action of the wave bed. The inclined angle of the wave bed makes the rock exit the separator with the help of pinch rollers.

A radial fan, mounted at the inlet of the duct system, provide the air to the air plenum. The fan, designed for 17,200 ACFM and a static pressure 9.8 inches of water, is driven by a 38.9 HP motor. This model of fan is currently used in most of the harvesting machines at Allan equipment. The primary use of such a high flow fan is to provide enough air to levitate potatoes above the wave bed. As the potatoes are levitated, the rocks stay at the bottom due to its higher density. The wave action of the belt therefore enables the separation of the rock at the side with help of pinch rollers. The fan is capable of functioning at different speeds with the help of Programmable Logic Controller and a Variable Frequency Drive. The research was carried out at an $80 \%$ load, which is the maximum load used when the harvester is used in the field.

In addition to the main fan, there is also a back fan which is connected to a rear duct that sends in air through ducts and into the pinch rollers present at the rock removal section. This back fan is used to make sure that the potatoes do not come out of the pinch rollers when the separation takes place.

The Wave-bed is a unique mechanism which is developed by Allan Equipment that generates a wave motion through a cam mechanism whose speed is also controlled. The belt is made up of steel rods coated with rubber which is thinly spaced. The portion below the belt consists of disks attached to two individual rods which move along an elliptical track and meet the wave bed along its width. This produces the wave like action on the bed.

Difficulty in separation was observed due to the uneven air flow distribution above the wave bed, which resulted in the clogging of potatoes at a few regions. This led to inefficient separation of the potatoes from rocks. The present research was made to achieve even air flow by using theoretical and experimental means.

\section{EXPERIMENTAL}

A series of experiments was conducted in a full scale commercial rock separator developed and used by Allan Equipment of Prince Edward Island. Primary objective of 
these experiment was to calibrate results of theoretical analysis and then check the results of some design improvements attempted to meet the requirements of this machine.

\subsection{Air flow measurement above the wave bed}

The region above the wave bed was analysed both theoretically and experimentally. Results of the theoretical analysis is discussed in section 3.1. Present section discusses the experimental results.

The purpose of this test was to understand the behavioural pattern of air flow above the wave bed. The testing was done by placing a Pitot tube above the wave bed and measuring the velocity \& pressure by connecting the Pitot tube to a digital manometer. The measurement was done by moving the instrument along the air plenum exit above the wave bed and recording the readings every six inches apart. The wave bed is shown in Fig 1.

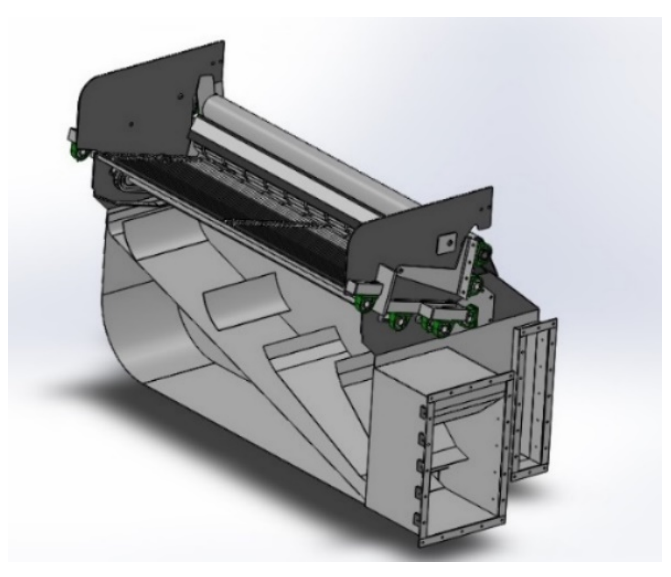

Fig 1 Design of the air plenum with baffles and wave bed

\subsection{Introduction of a Perforated plate}

Several attempts were made earlier to bring in flow uniformity by installing varied designs of flow straighteners in the air box, but they did not give the desired result. So, to achieve uniform air flow at the exit of the air plenum, a perforated sheet was introduced between the wave bed and the airbox exit. The velocity pressure at the exit of the wave bed was measured with the help of a pitot tube and a manometer. The initially used perforated plate was made up of $1 / 4$ inch holes equally spaced 1 inch apart throughout the entire area of 66x12 inches.

\subsection{Adjustment of hole size in perforated plate}

The velocity of the air escaping through the wave bed improved after the introduction of the perforated plate but it still appeared to uneven. So, an increase in the hole size in specific sections of low velocity was explored. The perforated plate was subdivided into 4 sections. These 4 sections were made into perforations of different sizes. (Fig 2)

\subsubsection{Layout 1}

This layout consists of 4 sections in which the section 1 is $3 / 8$ inch holes, the centre region has a mixture of alternate $1 / 4$ inch holes and $3 / 8$ inch holes and the section 3 is $1 / 2$ inch holes. There is an entire section of area 10x66 square inches section consisting of $1 / 4$ in holes.

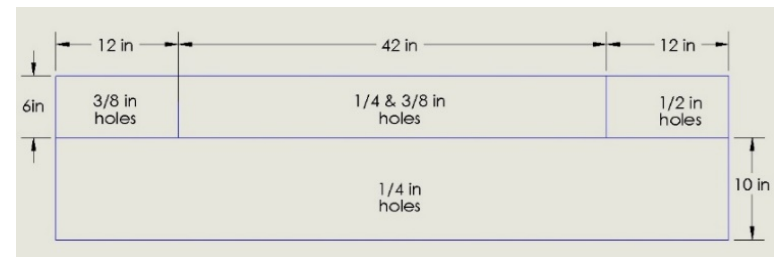

Fig 2 Layout 1 of the perforated plate

\subsection{Removal of the wave bed}

The wave bed was removed from the air plenum to measure the velocity directly above the perforated plate. Removing the wave bed allowed access for measuring the pressure difference across the perforated plate. The testing was done along the plate in the near end, middle and far end. The far end is the inlet of the potatoes. The perforated plate had the layout 1 sized holes (Fig 3).

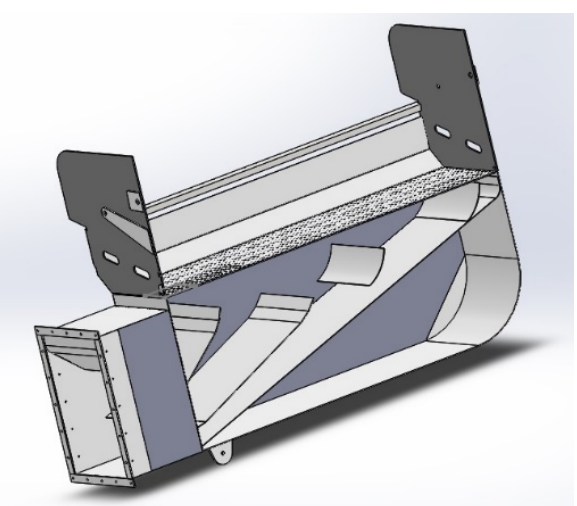

Fig 3 Perforated plate above the baffles with the wave bed removed

\subsection{Removal of the Baffles}

Now that uniformity of velocity distribution was attained mostly by the perforated plate the role of air box baffles or flow straighteners was limited. So, they were removed to examine its impact. An experiment was carried out after the baffles were removed (Fig 4). The layout of the perforation was the same as in Layout 1. (Fig 3)

The test was carried out in three regions; the near (To air entry) end, middle and far end. The Pitot tube was traversed across the perforated plate and the velocity pressure was measured.

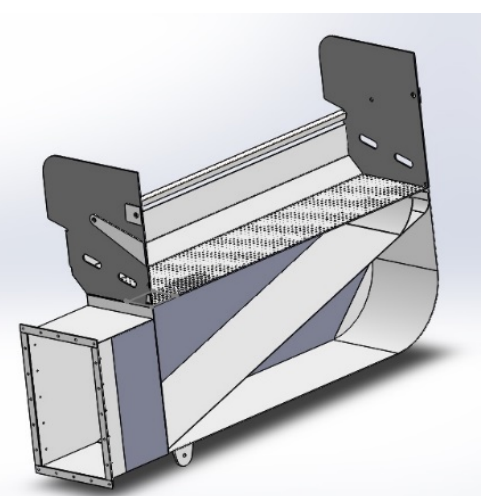

Fig 4 Perforated plate with both the baffles and wave bed removed 


\subsubsection{Factional opening for layout 1}

The fractional opening is important to understand the overall porosity of the perforated plate. This is calculated by dividing the total hole area by the total area.

Factional opening $=$ Total open area/ Total area

Table 1 Layout 1

\begin{tabular}{|l|r|r|r|}
\hline \multicolumn{4}{|c|}{ Factional opening calculation } \\
\hline & $\begin{array}{l}\text { Nos of } \\
\text { holes }\end{array}$ & $\begin{array}{l}\text { Hole size } \\
(\mathrm{mm})\end{array}$ & Area $\left(\mathrm{mm}^{2}\right)$ \\
\hline Section 1 & 72 & 9.52 & 5127 \\
\hline section 2 & 252 & 7.93 & 12467 \\
\hline section 3 & 72 & 12.7 & 9124 \\
\hline section 4 & 680 & 6.35 & 21543 \\
\hline
\end{tabular}

\begin{tabular}{|l|r|l|}
\hline Total void area & 48262 & $\mathrm{~mm}^{2}$ \\
\hline Plate Length & 1727 & $\mathrm{~mm}$ \\
\hline Plate Width & 406 & $\mathrm{~mm}$ \\
\hline Total Area & 701162 & $\mathrm{~mm}^{2}$ \\
\hline Total solid area & 652899 & $\mathrm{~mm}^{2}$ \\
\hline \multicolumn{3}{|c|}{} \\
\hline Fractional opening & $\mathbf{0 . 0 6 8}$ \\
\hline
\end{tabular}

\subsection{Increase in perforated plate hole size (Layout 2)}

The aim of the analysis being to increase the flow rate of air above the perforated plate, the hole size was increased further without changing the hole pattern. The hole size was increased to $40 \%$ of the layout 3 . The test was carried out in the presence of a flow meter to measure the flow rate of air through the fan suction inlet. The flow rate through the fan had reduced much from the previous test, carried out under similar fan load conditions. This reduction in flow rate could be attributed to the idea that the changes in weather conditions could have affected the density of air and as a result affecting fan performance.

The layout of the perforation is shown in Fig 5

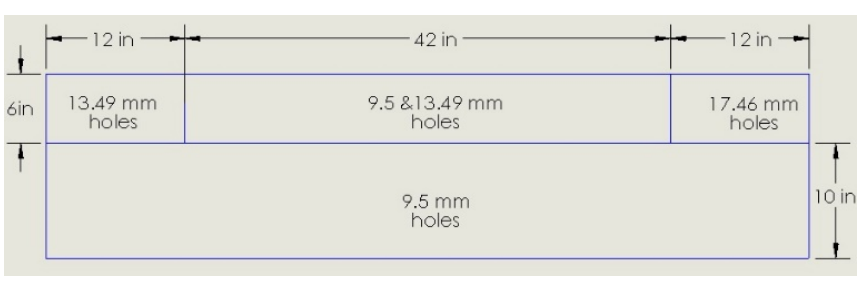

Fig 5 Layout 2

\subsubsection{Voidage for layout 2}

The Voidage of the perforated plate was calculated. This calculation is important to make sure that the hole size is not increased too much.

\begin{tabular}{|l|c|c|c|}
\hline & $\begin{array}{l}\text { no of } \\
\text { holes }\end{array}$ & $\begin{array}{l}\text { hole size } \\
(\mathrm{mm})\end{array}$ & Area $\left(\mathrm{mm}^{2}\right)$ \\
\hline Section 1 & 72 & 13.5 & 10294 \\
\hline section 2 & 252 & 11.5 & 26219 \\
\hline section 3 & 72 & 17.4 & 17249 \\
\hline section 4 & 680 & 9.5 & 48473 \\
\hline
\end{tabular}

\begin{tabular}{|l|r|l|}
\hline Total void area & 102237 & $\mathrm{~mm}^{2}$ \\
\hline Length & 1727 & $\mathrm{~mm}$ \\
\hline Width & 406 & $\mathrm{~mm}$ \\
\hline & & \\
\hline Total Area & 701162 & $\mathrm{~mm}^{2}$ \\
\hline Total solid area & 598924 & $\mathrm{~mm}^{2}$ \\
\hline Fractional opening & \multicolumn{2}{|c|}{$\mathbf{0 . 1 4 5}$} \\
\hline
\end{tabular}

\subsection{Attaching the wave bed}

When the potatoes are fed through the conveyor, they fall directly onto the wave bed shown in Fig 1. This wave bed is placed at a few inches above the perforated plate. So, it is ideal that we calculate the velocities above the wave bed and make desirable changes to the hole size in the perforated plate to make sure that the flow is even in the region above the wave bed. The wave bed is mounted at an inclination and of different sized rubbers wound upon each bar in the wave bed. This design of different size rubbers was to make the air more available at the rear end, as compared to the near end. The rear end of the wave bed is where the separation of the rocks takes place with the help of the pinch rollers, this region requires much air as compared to the near end. The movement of the potatoes to the outlet is mostly due the wave-like action of the bed, assisted by the slight bubbling provided by air.

\subsubsection{Layout 3}

The layout of the perforated plate was changed based on preliminary testing. This change in design was to provide more air flow towards the rear end and provide an even flow of air, so that there is no clogging up of clods in the regions above the wave bed. in Fig 6

The layout of the modified perforated plate is shown

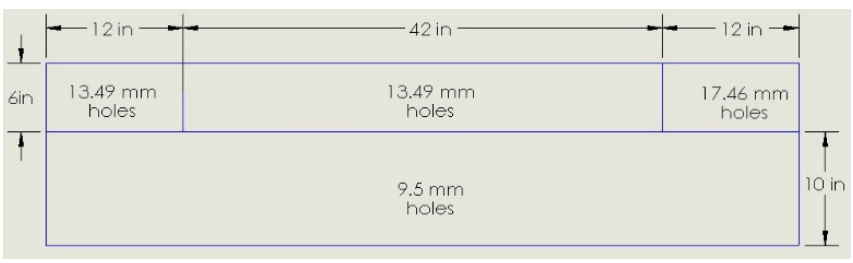

Fig 6 Layout 3

\subsubsection{Opening fraction for layout 3}

The Voidage calculation was done for Layout 5. This Voidage was done to make sure that the overall Porosity(or)Voidage doesn't exceed the nominal range of $20 \%$. 


\begin{tabular}{|l|c|c|r|}
\hline & $\begin{array}{l}\text { Nos of } \\
\text { holes }\end{array}$ & $\begin{array}{l}\text { Hole size } \\
(\mathrm{mm})\end{array}$ & Area $\left(\mathrm{mm}^{2}\right)$ \\
\hline Section 1 & 72 & 13.49 & 10295 \\
\hline section 2 & 252 & 13.49 & 36032 \\
\hline section 3 & 72 & 17.46 & 17250 \\
\hline section 4 & 680 & 9.52 & 48473 \\
\hline
\end{tabular}

\begin{tabular}{|l|r|l|}
\hline Total open area & 112050 & $\mathrm{~mm}^{2}$ \\
\hline Length & 1727 & $\mathrm{~mm}$ \\
\hline Width & 406 & $\mathrm{~mm}$ \\
\hline & & \\
\hline Total plate area & 701162 & $\mathrm{~mm}^{2}$ \\
\hline Total solid area & 589111 & $\mathrm{~mm}^{2}$ \\
\hline Opening fraction & \multicolumn{2}{|c|}{0.16} \\
\hline
\end{tabular}

\subsection{Removing the flow meter}

The velocity measurements were carried on the Layout 5 design without the presence of a flow meter. The isolation of the flow meter from the suction section of the fan is to record the velocity at which the harvester will function under ideal load conditions in the field.

\section{RESULTS AND DISCUSSION}

The velocities at regions above the wave bed was analysed using the CFD code of COMSOL whose results are shown in Figure 8a. These results were compared with those measured on the actual unit. Figure $8 \mathrm{~b}$ shows a reasonably good correlation between the flow predicted by CFD simulation and that measured. This verified validity of the present simulation.

\subsection{COMSOL CFD Results}

The simulation was also used to find the best possible design by using appropriate boundary condition. These boundary conditions were obtained by keeping the fan setting at initial load conditions and measuring the value of velocity with air exits from the centrifugal fan. Thus the need for hardware changes in the rock separator was avoided, thereby saving usage of raw materials and man power. The figures shown are for a few conditions in which the simulations results matched the physically conducted experiments.

Figure $8 \mathrm{~b}$ shows the region above the bed when the none of baffles, wave bed or perforated plate are present. This condition shows that without any resistance the velocity with which air exits the bed is concentrated at the back and not much at the fan inlet side. This design shows uneven air flow and hence there will be inefficient separation of potato. This simulation was, however, run at a lower average velocity of $33 \mathrm{~m} / \mathrm{s}$ as opposed to the nominal velocity with which the fan operates.

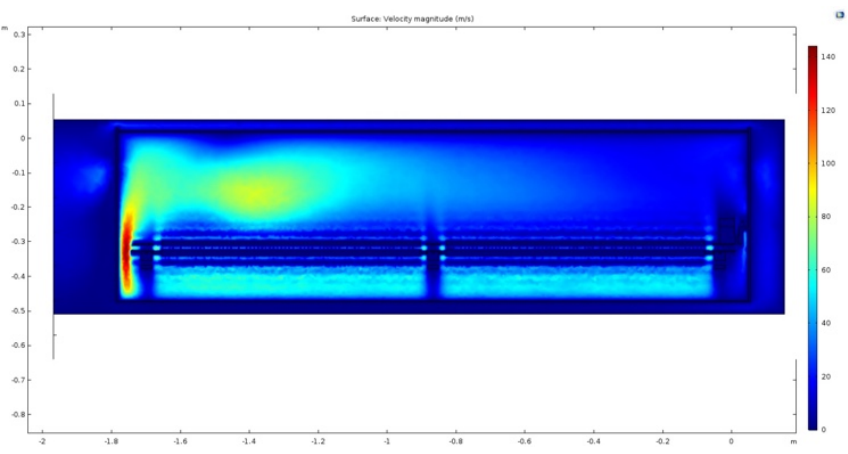

Fig 8 (a) Velocity above the wave bed with baffles and withoutt perforated plate as predicted by CFD.

Now to compare the measured results with the COMSOL simulation, they are plotted on Fig. 8b, which shows that the CFD obtained result closely resembles and matches with that measured at the rear end. Next simulation was carried out with both baffles and wave bed but without the perforated plate (Fig 8c). Based on the fan setting of 80 $\%$, the velocity of air coming through the inlet was kept at 43 $\mathrm{m} / \mathrm{s}$. Since there is no perforated plate, the velocity is high as indicated in the velocity scale. This pattern shows that in the centre region of the rear end, there is slight dip in the velocity.

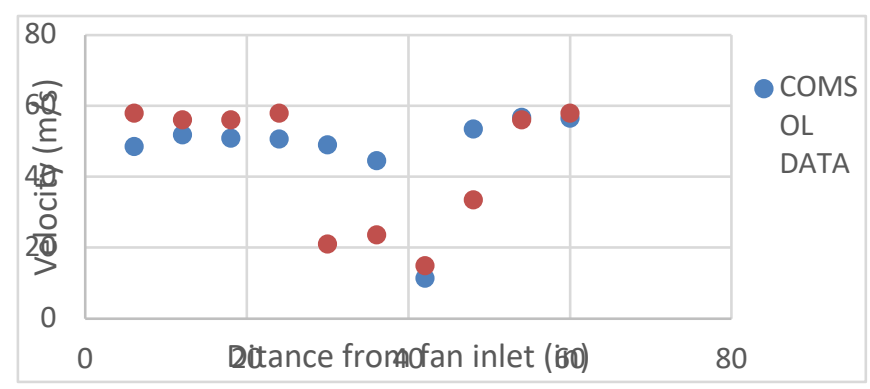

Fig $8 \mathrm{~b}$ comparison of velocity distribution as measured with that simulated using CFD above the air chamber with no perforated plate $\&$ baffles

A simulation was run for the layout 3 and this is compared with the experimental results in Fig 8(c) . Simulation results match the experimental results and shows an even distribution at the rear end. The velocity pattern is shown in Fig 8c. The analysis was carried out in the region above the perforated plate and hence the simulation's velocity profile is obtained at the region right above the perforated plate.

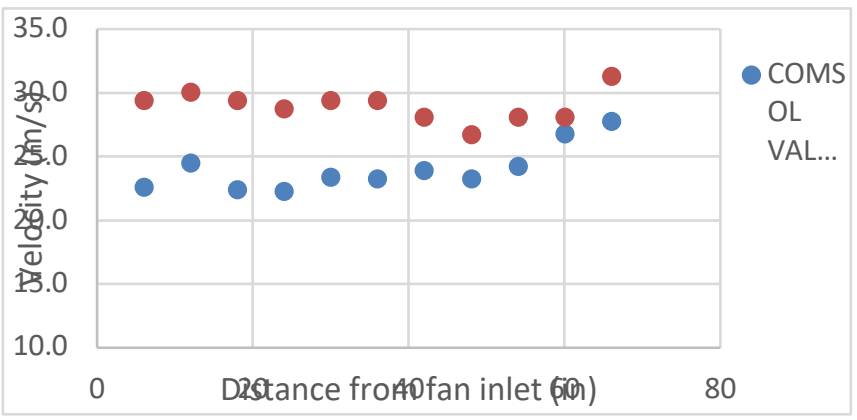

Fig 8c Comparison of Simulated velocity with those measured above the perforated plate without the baffles 


\subsection{Distance vs velocity for different conditions}

Case 1: Above wave bed-with baffles-without perforatedwithout flow meter-layout 1

The purpose of this test was to understand the behavioural pattern of air flow above the wave bed region in the air bed. The testing was done by placing a Pitot tube probe above the region and measuring the velocity pressure by connecting the Pitot tube to a digital manometer. The measurement was done by traversing the probe along the air plenum exit above the wave bed and recording the readings every six inches. The measurement was taken only at the rear end. In this experiment the baffles were present inside the air chamber, as shown in Fig 1.

From the readings obtained it can be inferred that the air velocity above the wave bed was not uniform. The centre region of the air plenum appears to have a significantly low velocity, shown in Fig 8b. COMSOL simulation was run with the required boundary conditions, and the obtained simulation result was fairly close to the experimental result, as shown in Fig 8b.

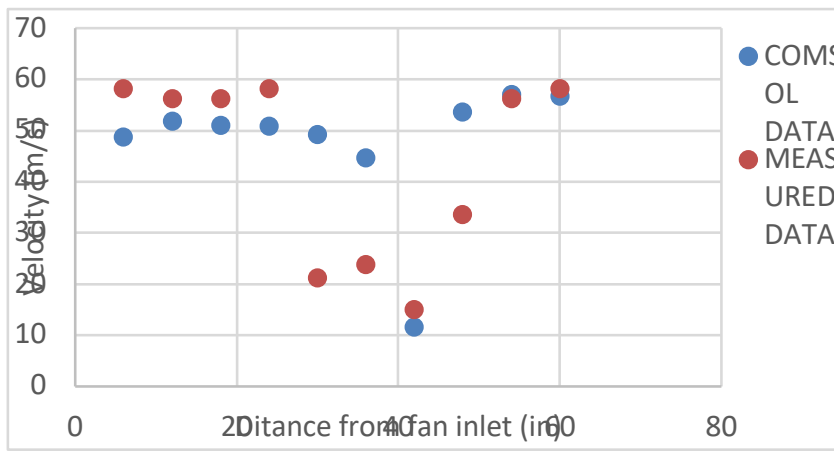

Fig 9 (a) Experimental and theoretical values of velocity plotted against distance from fan end of wave bed - case 1

Case 2: Above wave bed-with baffles-with perforated-without flow meter-layout 1

When the perforated plate of uniform holes was introduced in the air chamber, the flow was uniform in few regions but not everywhere, this was influenced by the design of the wave bed. After conducting initial testing on the air chamber, a change in design of the perforated plate was required. The initial testing was carried out on the updated design shown in Fig 3.2(b). The region above the wave bed was tested to find out the velocity pressure using a Pitot tube. The pitot tube was moved along the wave bed and the velocity pressure was measured.
Distance vs Velocity - Above wave bed with baffles \& Perfoarte

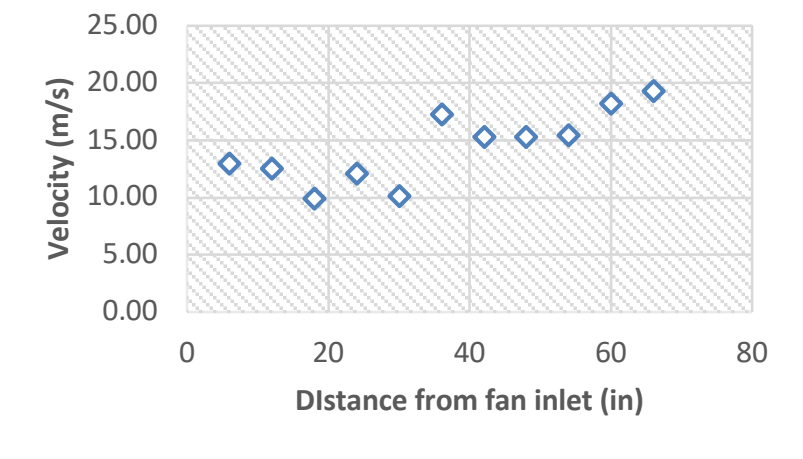

Fig 3.2(b) Experimental and theoretical velocity plotted against distance from fan end curve - case 2

Case 3: Above perforated-without baffles \& wave but with flow meter-Layout 1

The test was carried out again after removal of the baffles inside the airbox. The pitot tube measurements were taken at a height above the perforated plate and at distances long the place. A more uniform velocity distribution was loted after removal of the wave bed and baffles. The test was arried out in three regions: the near end, middle and far end. The pitot tube was traversed across the perforated plate and he velocity pressure was measured. The pressure difference cross the perforated plate was also measured and found to be $040 \mathrm{~Pa}$.

The velocity was more uniform above the perforated late compared with that measured above the wave bed (Fig ic).

A duct flowmeter of 12" diameter was attached at the suction section of the centrifugal fan and the flow rate was found to be 4834 CFM and 5.2 in of water, which reduced considerably from the original value of 17,200 ACFM.

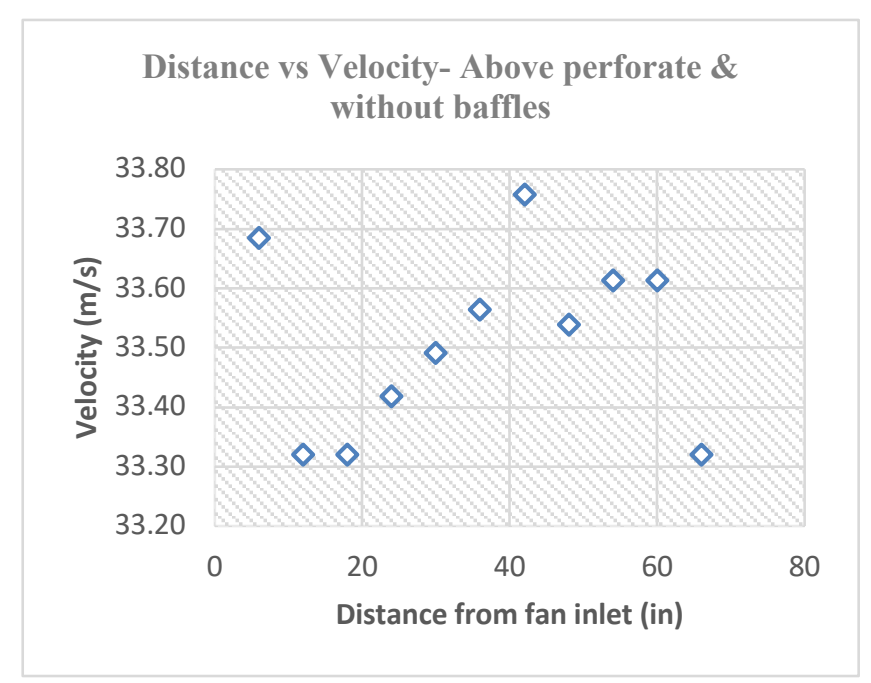

Fig 3.2(c) Distance vs Velocity above perforated plate without baffles case 3 
Case 4: Above perforated-without baffles-with flow meterlayout 2

The analysis being focused on improving the flow distribution of air above the perforated plate, the hole size was increased further without changing the hole pattern. The hole size was increased from that of the layout 1 . The bed pressure drop across the perforated plate was $600 \mathrm{~Pa}$. This test was carried out in the presence of a flow meter at suction inlet to measure the total flow rate of air through the fan. The flow rate through the fan had reduced much from the previous test, carried out under similar fan load conditions but without the flow meter. The fan was operated at the default load setting and the flow rate was measured to be $4834 \mathrm{cfm}$. This reduction in flow rate could be attributed to the high pressure drop (5.2 inch WG) across the flowmeter at fan inlet.

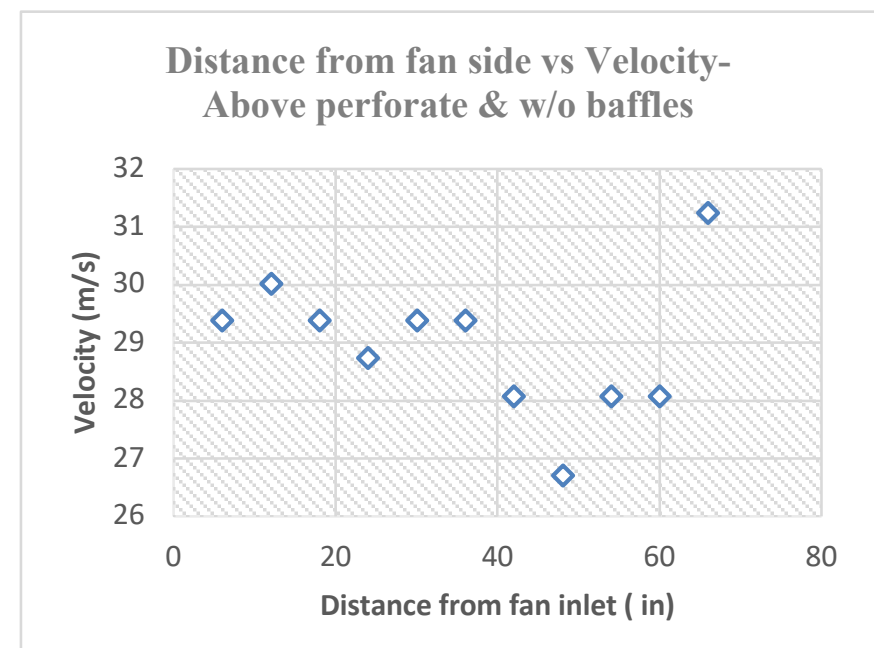

Fig 3.2(d) Distance vs Velocity above perforated plate without baffles- case

Case 5: Measurements above wave bed-without baffleswithout flowmeter- Layout 3

The velocity measurements were carried on the Layout 5 design without the presence of the duct flowmeter. The flowmeter was removed from the suction of the fan to measure the flowrate the harvester will have under actual operating conditions in the field. This showed no change in velocity distribution pattern but the magnitude of velocity had increased. Some drop in air velocity after passage through the wave bed is noted.

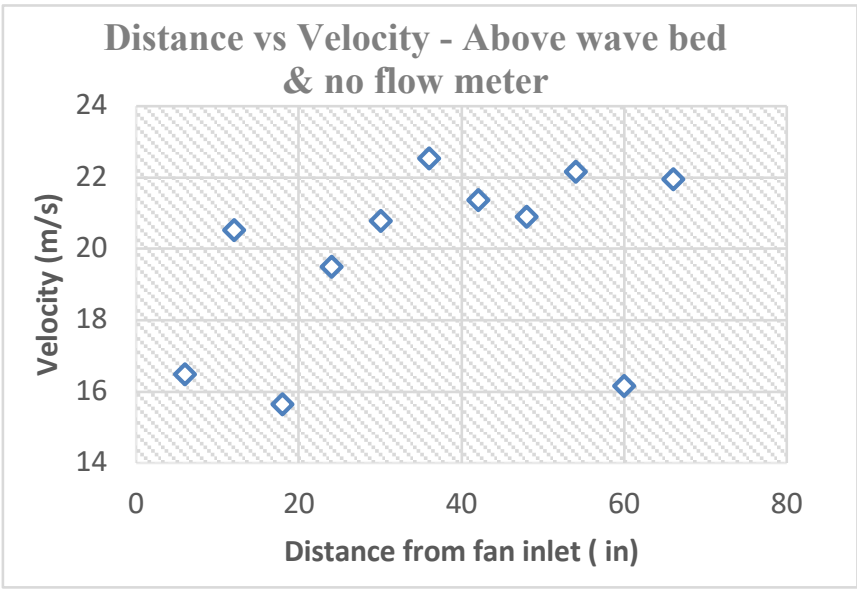

Fig 3.2(e) Distance vs Velocity above wave bed without flow meter- case 5

\section{CONCLUSION AND RECOMMENDATION}

The addition of a perforated plate in the airbox of the rock separator provided the rock separator with more even air distribution particularly in the desired sections of the separator. For simpler configuration a good agreement between velocities predicted by CFD with those measured experimentally. This validation allowed us to modify the air box design in the right direction without going through multiple trial and error. The design has, however, scope for further improvement. For example, the design of the wave bed can also be made more effective by including perforations right on the wave bed. 